\title{
Vitamin D Supplementation for Elderly Patient with DM Type 2 in Corona Virus Disease 19 Era
}

\author{
Putu Ayu Eramitha Harnumningrat D. ${ }^{1}$, Made Suma Wirawan ${ }^{2}$ \\ ${ }^{1,2}$ Department of Internal Medicine Wangaya Regional General Hospital Denpasar, Bali, Indonesia \\ Corresponding Author: Putu Ayu Eramitha Harnumningrat D.
}

DOI: https://doi.org/10.52403/ijrr.20220313

\begin{abstract}
Background and Purpose: Diabetes Mellitus is one of the commonest chronic metabolic diseases which has been increasing significantly, linked to obesity and advanced age, with high morbidity and mortality caused by its complication. On the other hand, vitamin D deficiency is also serious public health problem. In a recent year, vitamin $\mathrm{D}$ supplementation is recognised to have several roles on metabolic and glucose control, so are immune system. The aim of this study is focus on correlation between diabetes mellitus and vitamin $\mathrm{D}$, especially in elderly population, in the midst of global pandemic due to COVID-19. Method: The design of this study is literature review. A literature searching was carried out by using key term 'Diabetes Mellitus Type 2', 'Elderly Population', 'Vitamin D Supplementation', and 'COVID-19' from four database, including NCBI, PubMed, Science Direct, and Google Scholar.

Result: Administering vitamin D supplementation in elderly population with diabetes mellitus type 2 could have several benefits for improving $\mathrm{HbAlc}$, insulin resistance, metabolic profile, and blood glucose level. Moreover, vitamin D supplementation might help elderly patients with type 2 diabetes for having immunity to prevent infection, including COVID-19 infection.

Conclusion: Vitamin D supplementation can be considered as a simple and beneficial adjuvant therapy along with other treatment for diabetes mellitus in elderly population especially in this COVID-19 era.
\end{abstract}

Keywords: Diabetes Mellitus Type 2, Elderly, Vitamin D, Covid-19

\section{INTRODUCTION}

Type 2 Diabetes mellitus (T2DM) is one of the commonest chronic metabolic diseases in which incidence and prevalence has been increasing significantly in every country, linked to obesity and dyslipidaemia. It caused global health problem with high morbidity and mortality due to its complication and also decrease quality of life. Patient with T2DM had higher risk for microvascular complication such as neuropathy, nephropathy, and retinopathy and also macrovascular complication such as cardiovascular disease. Patient with T2DM especially who are having uncontrolled glycaemia status had a higher risk for acute and even lifethreatening complication such as diabetic ketoacidosis and hypoglycaemia.(Olokoba, et al, 2012; DeFronzo et al., 2015)

Several factors contribute to T2DM divided into environmental factors (obesity, unhealth diet, sedentary life, and physical inactivity), age, and genetic factors. These factors interacted one to another caused multiple pathophysiological disturbances that are responsible for impaired glucose homeostasis. The main mechanism in T2DM pathophysiology are insulin resistance and impaired insulin secretion in pancreas.(DeFronzo et al., 2015; Zheng,et al, 2018; Khan et al., 2020) Approximately in 2017 the prevalence of T2DM was 462 million individual, corresponding to $6.28 \%$ of the world population, or a prevalence rate of 6059 cases per 100.000 person. Mortality rate caused by T2DM alone was 1 million 
each year and making it the ninth leading cause of death globally. Increasing prevalence rate of obesity and another metabolic disease is similar to T2DM. Global prevalence of T2DM is projected to continue increase to 7079 individuals per 100.000 by 2030. And currently T2DM seem to emerging in young adult globally driven by the increasing burden of obesity and unhealthy life. (Song, 2012; Khan et al., 2020)

In elderly population, T2DM is becoming a serious public health concern. The elderly is a heterogenous group with different physiological profiles and varying functional capabilities and life expectancy. Morbidity and mortality due to T2DM complication are more frequent in old diabetic patient compared to the young adult. Geriatric problem such as eating disorder, lack physical activity, visual or memory trouble, and cognitive impairment which are usually appeared together with T2DM and other metabolic disease cause the management of T2DM for elderly population become more difficult and challenging. Thus, management of T2DM in elderly patients should be individualized. (Chentli, et al, 2015; Yakaryllmaz and Öztürk, 2017)

One of the adjuvant therapies which are currently recognized for T2DM patients is Vitamin D supplementation. Ioannis et al in his study concluded that vitamin D supplementation for T2DM patient has good impact on metabolic and lipid profile, oxidative stress, and complication of diabetes in older patients. In another hand, impairment of Vitamin D status in elderly is related to severe retinopathy, diabetic peripheral neuropathy, and poor cognition performance. (Papaioannou et al., 2021) Similar to Zhiweis et al's study about the efficacy of vitamin D supplementation on glycaemic control in type 2 diabetes patients, Zhiwei et al proved that vitamin D supplementation in T2DM patients could improve $\mathrm{HbAlc}$ and insulin resistance.(Hu et al., 2019). Especially in the era of Corona Virus Disease (COVID-19) pandemic which are occurred globally. The outbreak of COVID-19 has created public health problem that caused life-threatening infection or even death, particularly in highrisk population, included elderly with comorbid diseases. Preventive health measure that reduced risk of infection, progression, and severity are desperately needed. Vitamin D supplementation suggested possibly has a role in reducing risk of COVID-19 infection and the mortality caused by.(Ali, 2020; O'Shea et al., 2020; Singh, Jain and Singh, 2020)

\section{METHODS}

A comprehensive literature search was conducted on eligible electronic database: PubMed, NCBI, Science Direct, and Google Scholar by using keywords as follows: Elderly Population, Vitamin D supplementation, Type 2 Diabetes Mellitus, Corona Virus Disease, and COVID-19 outbreak. The search result from all databases is screened, evaluated, and analysed. The final inclusion of studies was determined by examining full article relating to the topic.

\section{RESULT AND DISCUSSION}

Diabetes Mellitus (DM) is one of chronic metabolic diseases with its main characteristic is persistent hyperglycaemia. Chronic hyperglycaemia occurred caused by impairment in insulin secretion and resistance to peripheral actions of insulin or could be both. Diabetes Mellitus is usually appeared synergy with other metabolic problems such as obesity, hypertension, and dyslipidaemia.

Currently, Diabetes Mellitus become global health problem with high morbidity and mortality due to its acute and chronic or microvascular and macrovascular complication leading to decrease quality of life. Diabetes mellitus, especially diabetes mellitus type 2 (T2DM) could affect various organ system and causes damage even lifethreatening event. (Goyal and Jialal, 2021) According to International Diabetes Federation (IDF), approximately 415 
million adults between the ages of 20 to 79 years suffering diabetes.(Zheng, Ley and $\mathrm{Hu}, 2018)$ T2DM become global epidemic which emerges rapidly. Several risk factors for developing T2DM are obesity, other chronic metabolic disease, unhealthy lifestyles, unhealthy diet, sedentary life, genetic, age, and others. (DeFronzo et al., 2015) Approximately in 2017 the prevalence of T2DM was 462 million individual, corresponding to $6.28 \%$ of the world population, or a prevalence rate of 6059 cases per 100.000 person. Mortality rate caused by T2DM alone was 1 million each year and making it the ninth leading cause of death globally. Increasing prevalence rate of obesity and another metabolic disease is similar to T2DM. Global prevalence of T2DM is projected to continue increase to 7079 individuals per 100.000 by 2030 . And currently T2DM seem to emerging in young adult globally driven by the increasing burden of obesity and unhealthy life. (Song, 2012; Khan et al., 2020) Complication of T2DM cause profound psychological and physical distress, not only for patient but for family and put a huge burden disease to health-care system.(Chatterjee, Khunti and Davies, 2017)

The risk of diabetes is increasing with age. In general, about $20 \%$ of old people have DM and a similar proportion have undiagnosed DM. Elderly could have different symptoms compared to common clinical symptoms of T2DM. The disease ay either be less visible or differ from typical ones, and even can be asymptomatic until it causes several complications. Some clinical symptoms, such as polydipsia doesn't occur in elderly and along with osmotic diuresis, it may cause serious dehydration and often requiring hospitalization. Additionally, increase with age, risk factor to develop other chronic metabolic disease is also increasing accompanied by decreasing various organ system function, included pancreas and other age-associated disease like memory disorders, general malaise, eating disorder, apathy, mental confusion, dysphoria, and dementia. Risk for developing both acute and chronic complication of T2DM is also higher in elderly. The vulnerability to hypoglycaemia is substantially increased in older adults. (Chentli, Azzoug and Mahgoun, 2015; Rymkiewicz et.al., 2015) Thus, management of T2DM in elderly patients could be challenging and should be individualized. (Chentli, et al, 2015; Yakaryılmaz and Öztürk, 2017)

In elderly population, Vitamin D deficiency seems to be an additional risk factor linked to higher risk of osteoporosis, insulin resistance, obesity, cognitive impairment, and Alzheimer disease. (Chentli, Azzoug and Mahgoun, 2015) Ioannis et al in their study showed strong correlation between Vitamin D Deficiency and risk for developing Type 2 Diabetes Mellitus in elderly, included the severity of the symptoms and its complication. (Papaioannou et.al. 2021)Therefore, recently, researchers seem to recognize Vitamin D Supplementation as one of the adjunctive therapies of T2DM, especially in elderly population.

Vitamin D3 (cholecalciferol) is the natural form of vitamin $\mathrm{D}$, produced in the skin from 7-dehydrocholesterol under the influence of UV light. Metabolism of vitamin D involved liver, kidney and skin. (Seshadri, Tamilselvan and Rajendran, 2011)In recent years, Vitamin D has become interesting with its impact on multiple biology processes including inhibition of various cancer progression, effect on cardiovascular system, and protection against a number of autoimmune diseases. (Christakos et al., 2016; Bikle, 2017) Vitamin D is mainly important for bone and mineral metabolism. But since vitamin D receptors (VDRs) are expressed in almost every tissue and cell, it seems to have potential extra-skeletal effect on various organ system, including endocrine system.(Pilz et al., 2019)

Tang et al in his study concluded that administering vitamin D supplementation for T2DM patients might 
improve glucose and insulin metabolism and has no effect on developing diabetes mellitus among nondiabetic adults. This study showed that vitamin D supplementation significantly decrease fasting glucose level and improve insulin homeostasis. Several pharmacological mechanisms have been proposed to explain how vitamin D could have impact and regulate metabolism of glucose and insulin. Vitamin D homeostasis suggested has an essential role for both insulin sensitivity and secretion by directly stimulates insulin receptors to enhance insulin sensitivity and insulin responsiveness for glucose transport in cell and vascular. Indirect mechanism of vitamin D effect on insulin resistance is through adiposity cells. Moreover, vitamin $\mathrm{D}$ affects the release of insulin via binding to the vitamin D receptor in Beta cell pancreas and vitamin $\mathrm{D}$ dependent calciumbinding proteins. Through $\beta$-cells via regulation of calcium transport, vitamin D indirectly impact calcium transport. Some sources showed the decreasing of fasting glucose level and HbA1C after T2DM patient taking vitamin D supplementation, especially for ages 71 years and older.(Tang, Hartz and Bauer, 2017; Maddaloni et al., 2018)

Similar to systematic review and meta-analysis by Yvonne et al which concluded that vitamin D supplementation has favourable effect in improving fasting glucose level. Vitamin D receptor (VDR) could be found in most cell, including the pancreatic beta-cells. Vitamin D is also well-known to have immune-modulatory and anti-inflammatory effects and through this mechanism might lead to improve peripheral insulin resistance by altering lowgrade chronic inflammation.(Krul-Poel et al., 2017) It tends to down-regulate the transcriptions of various pro-inflammatory cytokine genes like interleukin-2, interleukin-12, and Tumour Necrosis Factor-alpha. It also has a role to promote the induction of regulatory T-lymphocytes, the production of anti-inflammatory cytokines and protects beta-cell from destruction. (Seshadri, Tamilselvan and Rajendran, 2011) Vitamin D concentration level is relating to how high the risk of T2DM patient might have complication from diabetes mellitus, especially cardiovascular events and renal impairment. (Palomer et al., 2008; Seshadri et al., 2011; Zakhary et al., 2021)

Exclusive in elderly population with T2DM, vitamin D deficiency become serious health problem with significant impact and multiple health effects. Risk factor for vitamin D deficiency in elderly was malabsorption, reduced renal function, skin decline, reduced time outdoors, and interaction of multiple pharmacies. Old people with impairment of vitamin D level have a higher risk suffering cognitive problem, depression, osteoporosis, cardiovascular disease, hypertension, cancer, and also diabetes mellitus. Both of T2DM and vitamin D deficiency are interacting one to each other and cause worsening condition. The main pathophysiology mechanism relating to DM and vitamin D deficiency are dysregulated of pancreatic beta-cell function, impaired insulin sensitivity, and increased systemic inflammation.(Papaioannou et al., 2021)

Since vitamin D has many impacts on health problem, especially in elderly population, many scientists recognized the benefit of administering vitamin D supplementation for elderly population with T2DM. Papaiounnou et al in his study revealed that therapeutic regiment of vitamin D supplementation could improve endothelial function, increased insulin sensitivity and beta-cell pancreas function, reduced oxidative stress, and improved metabolic parameter to elderly with T2DM. Moreover, high dose of vitamin D supplementation in T2DM patients could improve blood pressure and b-type natriuretic peptide level. Status of vitamin D sufficiency level is associated with higher self-reported diabetes treatment satisfaction and better diabetes-specific quality of life in elderly population.(Papaioannou et al., 2021) Moreover in the midst of global 
pandemic due to COVID-19, viral infection caused by a novel beta coronavirus, wellknown as Sever Acute Respiratory Syndrome-Coronavirus-2 (SARS-CoV-2). Diabetes Mellitus, advanced age, and also vitamin $\mathrm{D}$ deficiency have been recognised as a risk factor for severe clinical feature of COVID-19 infection and increase mortality and hospitalization in COVID-19 infection. These risk factors are also increasing the susceptibility to have infection. Impaired immune response to infection leading to alteration of cytokine profile and changes in immune system including T-cell and macrophage activation. Poor glycaemic control and immunocompromised condition are also leading to secondary various infection including bacterial, parasitic, and mycotic infection. Therefore, adjuvant therapy, especially vitamin D supplementation suggested to have beneficial effect for elderly patient with T2DM. (Singh, Jain and Singh, 2020)

\section{CONCLUSION}

In summary, administering vitamin D supplementation in elderly population with diabetes mellitus type 2 could have several benefits for improving $\mathrm{HbAlc}$, insulin resistance, metabolic profile, and blood glucose level. Moreover, vitamin D supplementation might help elderly patients with type 2 diabetes for having immunity to prevent infection, including COVID-19 infection. Therefore, vitamin $\mathrm{D}$ can be considered as a simple adjuvant therapy along with other treatment for diabetes mellitus.

\section{DECLARATION}

Acknowledgement: None

\section{Conflict of Interest: None}

Source of Funding: This work did not receive any grant from funding agencies in the public, commercial, or not for profit sector

Ethical approval: Not required

\section{REFERENCES}

1. Ali, N. Role of vitamin D in preventing of COVID-19 infection, progression and severity. Journal of Infection and Public Health. 2020; 13(10): 1373-1380. doi:10.1016/j.jiph.2020.06.021.

2. Bikle, D. Vitamin D: Production, Metabolism, and Mechanisms of Action. Chemical Biology Journal.2014; 21(3); 319329. DOI: 10.1016/j.chembiol.2013.12.016

3. Chatterjee, S., Khunti, K. and Davies, M.J. Type 2 diabetes. The Lancet. 2017; 389(10085): 2239-2251. doi:10.1016/ S0140-6736(17)30058-2.

4. Chentli, F., Azzoug, S. and Mahgoun, S. Diabetes mellitus in elderly. Indian Journal of Endocrinology and Metabolism. 2015; 19(6): 744-752. doi:10.4103/22308210.167553.

5. Christakos, S. et al. Vitamin D: Metabolism, Molecular Mechanism of Action, and Pleiotropic Effects. Physiological Reviews. 2016; 96(1): 365-408. doi:10.1152/physrev. 00014.2015.

6. DeFronzo, R.A. et al.Type 2 diabetes mellitus. Nature Reviews Disease Primers. 2015;1(1): 1-22. doi:10.1038/nrdp.2015.19.

7. Goyal, R. and Jialal, I. Diabetes Mellitus Type 2. StatPearls Publishing 2021. Available at: https://www.ncbi.nlm.nih.gov/books/NBK5 13253/ (Accessed: 24 February 2022).

8. $\mathrm{Hu}, \mathrm{Z}$. et al. Efficacy of vitamin D supplementation on glycemic control in type 2 diabetes patients. Medicine. 2019; 98(14): e14970.doi:10.1097/MD.000000000001497 0.

9. Khan, M.A.B. et al. Epidemiology of Type 2 Diabetes-Global Burden of Disease and Forecasted Trends. Journal of Epidemiology and Global Health. 2020; 10(1): pp. 107111. doi:10.2991/jegh.k.191028.001.

10. Krul-Poel, Y.H.M. et al. Management of endocrine disease: The effect of vitamin D supplementation on glycaemic control in patients with type 2 diabetes mellitus: a systematic review and meta-analysis. European Journal of Endocrinology. 2019; 176(1): R1-R14. doi:10.1530/EJE-16-0391.

11. Maddaloni, E. et al. Vitamin D and Diabetes Mellitus.Vitamin D in Clinical Medicine. 2018; 50: 161-176. doi:10.1159/000486083.

12. Olokoba, A.B., Obateru, O.A. and Olokoba, L.B. Type 2 Diabetes Mellitus: A Review of Current Trends. Oman Medical Journal. 
Putu Ayu Eramitha Harnumningrat D. et.al. Vitamin D supplementation for elderly patient with DM type 2 in Corona Virus Disease 19 Era

2012; 27(4): 269-273. doi:10.5001/omj. 2012.68.

13. O'Shea, P.M. et al.COVID-19: the older adult and the importance of vitamin $\mathrm{D}$ sufficiency. Journal of Nutritional Science. 2020; 9: e40. doi:10.1017/jns.2020.36.

14. Palomer, X. et al. Role of vitamin D in the pathogenesis of type 2 diabetes mellitus. Diabetes, Obesity \& Metabolism. 2008; 10(3): 185-197. doi:10.1111/j.1463-1326. 2007.00710.x.

15. Papaioannou, I. et al. Vitamin D Deficiency in Elderly with Diabetes Mellitus Type 2: A Review. Cureus. 2021; 13(1). doi:10.7759/ cureus. 12506.

16. Pilz, S. et al. Vitamin D testing and treatment: a narrative review of current evidence. Endocrine Connections. 2019; 8(2): R27-R43. doi:10.1530/EC-18-0432.

17. Rymkiewicz, E. et al. Diabetes mellitus type 2 in the elderly. Polish Journal of Public Health. 2015; 125. doi:10.1515/pjph-20150021.

18. Seshadri, K.G., Tamilselvan, B. and Rajendran, A. Role of Vitamin D in Diabetes. Journal of Endocrinology and Metabolism. 2011; 1(2): 47-56. doi: 10.4021/jem.v1i2.23.

19. Singh, S.K., Jain, R. and Singh, S. Vitamin $\mathrm{D}$ deficiency in patients with diabetes and COVID- 19 infection. Diabetes \& Metabolic
Syndrome. $\quad 2020 ; \quad 14(5): \quad$ 1033-1035. doi:10.1016/j.dsx.2020.06.071.

20. Song, S.H. Emerging type 2 diabetes in young adults. Advances in Experimental Medicine and Biology. 2012; 771: 51-61. doi:10.1007/978-1-4614-5441-0_7.

21. Tang, F., Hartz, A.M.S. and Bauer, B.DrugResistant Epilepsy: Multiple Hypotheses, Few Answers', Frontiers in Neurology. 2017; 8. doi:10.3389/fneur.2017.00301.

22. Yakary1lmaz, F.D. and Öztürk, Z.A. Treatment of type 2 diabetes mellitus in the elderly. World Journal of Diabetes. 2017; 8(6): 278-285. doi:10.4239/wjd.v8.i6.278.

23. Zakhary, C.M. et al. Protective Role of Vitamin D Therapy in Diabetes Mellitus Type II.Cureus. 2021; 13(8). doi:10.7759/ cureus.17317.

24. Zheng, Y., Ley, S.H. and Hu, F.B. Global aetiology and epidemiology of type 2 diabetes mellitus and its complications. Nature Reviews Endocrinology. 2018; 14(2): 88-98. doi:10.1038/nrendo.2017.151.

How to cite this article: Putu Ayu Eramitha Harnumningrat D., Made Suma Wirawan. Vitamin D supplementation for elderly patient with DM type 2 in Corona Virus Disease 19 Era. International Journal of Research and Review. 2022; 9(3): 99-104. DOI: https:// doi.org/10.52403/ijrr.20220313 